\title{
Eigenrelations for Nonviscously Damped Systems
}

\author{
Sondipon Adhikari* \\ University of Cambridge, Cambridge, England CB2 1PZ, United Kingdom
}

\begin{abstract}
Multiple-degree-of-freedom linear dynamic systems with nonviscous damping is considered. The nonviscous damping is such that the damping forces depend on the past history of the velocities via convolution integrals over some kernel functions. For the sake of generality, the system matrices are allowed to be asymmetric. The mode-orthogonality relationships, known for undamped or viscously damped systems, have been generalized to such nonviscously damped systems. Some expressions are suggested for the normalization of the eigenvectors. A number of useful results relating the system matrices and the eigensolutions have been established. Numerical examples are provided to illustrate the derived results.
\end{abstract}

\section{Nomenclature}

$=$ viscous damping matrix

$=$ space of complex numbers

$=$ dynamic stiffness matrix

$=$ damping function in the Laplace domain

$=$ damping function in the time domain

$=$ identity matrix of size $N$

$=$ unit imaginary number, $i=\sqrt{ }-1$

$=$ stiffness matrix

$=$ mass matrix

$=$ order of the characteristic polynomial

$=$ degrees of freedom of the system

= null matrix of size $N$

number of nonviscous modes

space of real numbers

Laplace domain parameter

= time

matrices of the right and left eigenvectors

Laplace transform of $\mathfrak{u}(t)$

response vector

Dirac-delta function

$=$ Kroneker-delta function

$=$ normalization matrix

partition of $\Theta$

$=$ diagonal matrix containing $\lambda_{k}$

partition of $\Lambda$

$=k$ th eigenvalue of the system

$=$ parameters of the GHM (Golla, McTavis, and Hughes method) damping model

$=$ diagonal matrix containing $v_{k}(s)$

$=k$ th eigenvalue of $\boldsymbol{D}(s)$

$\Phi(s), \Psi(s)=$ matrices of the right and left eigenvectors of $\boldsymbol{D}(s)$

$\omega_{k} \quad=k$ th undamped natural frequency

$\|\bullet\| \quad=\quad l_{2}$ matrix norm

Subscripts

e $\quad=$ elastic modes

$n \quad=$ nonviscous modes
Superscripts

$-1$

$-1$

$(\cdot)$

$(*)$

$=$ matrix transposition
$=$ matrix inversion
$=$ differentiation with respect to $t$
$=$ differentiation with respect to $s$
$=$ complex conjugation

Received 1 July 2000; revision received 5 February 2001; accepted for publication 8 February 2001. Copyright (C) 2001 by the American Institute of Aeronautics and Astronautics, Inc. All rights reserved.

* Research Associate, Department of Engineering, Trumpington Street; sa225@eng.cam.ac.uk.

\section{Introduction}

$\mathbf{P}$ OSSIBLY the most general way to model damping within the linear range is to consider nonviscous damping models that depend on the past history of motion via convolution integrals over some kernel functions. The equations of motion describing free vibration of an $N$-degree-of-freedom linear system with such damping can be expressed by

$$
\boldsymbol{M} \ddot{\mathfrak{u}}(t)+\int_{-\infty}^{t} \mathcal{G}(t-\tau) \dot{\mathfrak{u}}(\tau) \mathrm{d} \tau+\boldsymbol{K} \mathfrak{u}(t)=\mathbf{0}
$$

Here $\boldsymbol{M}$ and $\boldsymbol{K} \in \mathbb{R}^{N \times N}$ are the mass and stiffness matrices, $\mathcal{G}(t) \in \mathbb{R}^{N \times N}$ is the matrix of kernel functions, and $\mathbf{0}$ is an $N \times 1 \mathrm{vec}-$ tor of zeros. In the special case when $\mathcal{G}(t-\tau)=\boldsymbol{C} \delta(t-\tau)$, Eq. (1) reduces to the case of viscously damped systems. The damping model of this kind is a further generalization of the familiar viscous damping. Recently Adhikari ${ }^{1}$ has proposed a method to obtain the eigenvalues, eigenvectors, and dynamic response of such systems. The purpose of this paper is to develop some relationships satisfied by the eigensolutions and the system matrices of Eq. (1).

\section{Eigensolutions}

Taking the Laplace transform of Eq. (1), we have

$$
s^{2} \boldsymbol{M} \overline{\boldsymbol{u}}+s \boldsymbol{G}(s) \overline{\boldsymbol{u}}+\boldsymbol{K} \overline{\boldsymbol{u}}=\mathbf{0} \quad \text { or } \quad \boldsymbol{D}(s) \overline{\boldsymbol{u}}=\mathbf{0}
$$

where the dynamic stiffness matrix

$$
\boldsymbol{D}(s)=s^{2} \boldsymbol{M}+s \boldsymbol{G}(s)+\boldsymbol{K} \in \mathbb{C}^{N \times N}
$$

Here $\overline{\boldsymbol{u}}(s)=\mathcal{L}[\boldsymbol{u}(t)] \in \mathbb{C}^{N}, \boldsymbol{G}(s)=\mathcal{L}[\mathcal{G}(t)] \in \mathbb{C}^{N \times N}$, and $\mathcal{L}[\bullet]$ denotes the Laplace transform. In the context of structural dynamics, $s=i \omega$, where $\omega \in \mathbb{R}^{+}$denotes the frequency. To achieve generality, we allow the system matrices to be asymmetric, and also no assumption has been made regarding the positive definiteness of the system matrices. We consider the damping to be nonproportional, that is, the mass and stiffness matrices as well as the matrix of the kernel functions cannot be simultaneously diagonalized by any linear transformation. However, it is assumed that $\boldsymbol{M}^{-1}$ exists and $\boldsymbol{G}(s)$ is such that the motion is dissipative. Conditions that $\boldsymbol{G}(s)$ must satisfy in order to produce dissipative motion were given by Golla and Hughes. ${ }^{2}$ They have also given several physically realistic mathematical forms of the elements of $\boldsymbol{G}(s)$ available in the literature. Here, we do not assume any specific functional form of $G_{j k}(s)$ but assume that $\left|G_{j k}(s)\right|<\infty$ when $s \rightarrow \infty$. This in turn implies that the elements of $\boldsymbol{G}(s)$ are at the most of order $1 / s$ in $s$ or constant, as in the case of viscous damping. The eigenvalues $\lambda_{j}$, associated with Eq. (2), are roots of the characteristic equation

$$
\operatorname{det}\left[s^{2} \boldsymbol{M}+s \boldsymbol{G}(s)+\boldsymbol{K}\right]=0
$$

Suppose the order of the characteristic equation is $m$. In general $m$ is more than $2 N$, that is, $m=2 N+p ; p \geq 0$. Thus, although the 
system has $N$ degrees of freedom, the number of eigenvalues is more than $2 N$. This is a major difference between the nonviscously damped systems and the viscously damped systems where the number of eigenvalues is exactly $2 N$, including any multiplicities. It is assumed that all $m$ eigenvalues are distinct. Construct the diagonal matrix containing the eigenvalues as

$$
\Lambda=\operatorname{diag}\left[\lambda_{1}, \lambda_{2}, \ldots, \lambda_{m}\right] \in \mathbb{C}^{m \times m}
$$

The right and left eigenvalue problems associated with Eq. (1) can be defined from Eq. (2) as

$$
\begin{array}{ll}
{\left[\lambda_{j}^{2} \boldsymbol{M}+\lambda_{j} \boldsymbol{G}\left(\lambda_{j}\right)+\boldsymbol{K}\right] \boldsymbol{u}_{j}=\mathbf{0},} & \forall j=1, \ldots, m \\
\boldsymbol{v}_{k}^{T}\left[\lambda_{k}^{2} \boldsymbol{M}+\lambda_{k} \boldsymbol{G}\left(\lambda_{k}\right)+\boldsymbol{K}\right]=\mathbf{0}^{T}, & \forall k=1, \ldots, m
\end{array}
$$

Here $\boldsymbol{u}_{j}$ is the $j$ th right eigenvector and $\boldsymbol{v}_{k}$ is the $k$ th left eigenvector. These eigenvalue problems are not similar to the eigenvalue problems arise in the context of Lambda matrices ${ }^{3}$ because $\boldsymbol{G}(s)$ is not a constant matrix. Define the matrices of the right and left eigenvectors (the modal matrices) as

$$
\begin{aligned}
& \boldsymbol{U}=\left[\boldsymbol{u}_{1}, \boldsymbol{u}_{2}, \ldots, \boldsymbol{u}_{m}\right] \in \mathbb{C}^{N \times m} \\
& \boldsymbol{V}=\left[\boldsymbol{v}_{1}, \boldsymbol{v}_{2}, \ldots, \boldsymbol{v}_{m}\right] \in \mathbb{C}^{N \times m}
\end{aligned}
$$

If the damping is light, then among the $m$ eigenvalues $2 N$ appear in complex conjugate pairs. ${ }^{1}$ Under such assumptions it is easy to show that the rest $p=m-2 N$ eigenvalues become purely real. Corresponding to the $2 \mathrm{~N}$ complex conjugate pairs of eigenvalues, the $N$ right and left eigenvectors together with their complex conjugates are called elastic modes. These modes are related to the $N$ modes of vibration of the structural system. Physically, " $2 N$ complex conjugate pairs of eigenvalues" implies that all of the elastic modes are oscillatory in nature, that is, they are subcritically damped. The modes corresponding to the additional $p$ eigenvalues are called nonviscous modes. These modes are induced by the nonviscous effect of the damping mechanism. For stable passive systems the nonviscous modes are overcritically damped (i.e., negative real eigenvalues) and not oscillatory in nature. Next, we consider the normalization relationship of the eigenvectors.

\section{Normalization of the Eigenvectors}

Premultiplying Eq. (6a) by $\boldsymbol{v}_{k}^{T}$, postmultiplying Eq. (6b) by $\boldsymbol{u}_{j}$, and subtracting one from the other, we obtain

$$
\boldsymbol{v}_{k}^{T}\left[\left(\lambda_{j}^{2}-\lambda_{k}^{2}\right) \boldsymbol{M}+\lambda_{j} \boldsymbol{G}\left(\lambda_{j}\right)-\lambda_{k} \boldsymbol{G}\left(\lambda_{k}\right)\right] \boldsymbol{u}_{j}=0
$$

Because $\lambda_{j}$ and $\lambda_{k}$ are distinct for different $j$ and $k$, the preceding equation can be divided by $\left(\lambda_{j}-\lambda_{k}\right)$ to obtain

$$
\begin{array}{r}
\boldsymbol{v}_{k}^{T}\left[\left(\lambda_{j}+\lambda_{k}\right) \boldsymbol{M}+\frac{\lambda_{j} \boldsymbol{G}\left(\lambda_{j}\right)-\lambda_{k} \boldsymbol{G}\left(\lambda_{k}\right)}{\lambda_{j}-\lambda_{k}}\right] \boldsymbol{u}_{j}=0 \\
\forall j, k ; \quad j \neq k
\end{array}
$$

This equation can be regarded as the orthogonality relationship of the eigenvectors. It is easy to verify that in the undamped limit Eq. (10) degenerates to the familiar mass orthogonality relationship of the undamped eigenvectors. However, this orthogonality relationship is not very useful because it is expressed in terms of the natural frequencies. A frequency-independent orthogonality relationship of the eigenvectors will be derived later in this paper. Assuming $\delta_{\lambda}=\lambda_{j}-\lambda_{k}$, rewrite Eq. (10) as

$$
\boldsymbol{v}_{k}^{T}\left[\left(\delta_{\lambda}+2 \lambda_{k}\right) \boldsymbol{M}+\frac{\left(\lambda_{k}+\delta_{\lambda}\right) \boldsymbol{G}\left(\lambda_{k}+\delta_{\lambda}\right)-\lambda_{k} \boldsymbol{G}\left(\lambda_{k}\right)}{\delta_{\lambda}}\right] \boldsymbol{u}_{j}=0
$$

Consider the case when $\lambda_{j} \rightarrow \lambda_{k}$, that is, $\delta_{\lambda} \rightarrow 0$. Substituting $\lambda_{j}=\lambda_{k}$ in Eq. (9), it is easy to verify that when the right-hand side is zero the relationship represents a trivial case. For a nontrivial case the right hand-side of Eq. (9) must be nonzero as $\lambda_{j} \rightarrow \lambda_{k}$. So, for $\delta_{\lambda} \rightarrow 0$ Eq. (11) reads

$$
\boldsymbol{v}_{k}^{T}\left[2 \lambda_{k} \boldsymbol{M}+\left.\frac{\partial[s \boldsymbol{G}(s)]}{\partial s}\right|_{\lambda_{k}}\right] \boldsymbol{u}_{k}=\theta_{k}
$$

or

$$
\boldsymbol{v}_{k}^{T}\left[2 \lambda_{k} \boldsymbol{M}+\boldsymbol{G}\left(\lambda_{k}\right)+\lambda_{k} \boldsymbol{G}^{\prime}\left(\lambda_{k}\right)\right] \boldsymbol{u}_{k}=\theta_{k}
$$

$\forall k=1, \ldots, m$ for some nonzero $\theta_{k} \in \mathbb{C}$. Equation (13) is the normalization relationship for the right and left eigenvectors of the nonviscously damped system (1). From the expression of the dynamic stiffness matrix in Eq. (3), the normalization condition in Eq. (13) can also be expressed as

$$
\boldsymbol{v}_{k}^{T} \boldsymbol{D}^{\prime}\left(\lambda_{k}\right) \boldsymbol{u}_{k}=\theta_{k}, \quad \forall k=1, \ldots, m
$$

Equation (13), and consequently Eq. (14), can be regarded as the generalization of the "mass normalization" relationship used in structural dynamics. In the undamped limit when $\boldsymbol{G}(s)$ is a null matrix, Eq. (13) reduces to the familiar mass normalization relationship for the undamped eigenvectors. For viscously damped systems (see the Appendix for details), a relationship analogous to Eq. (13) was obtained, for example, by Sestieri and Ibrahim ${ }^{4}$ using state-space approach and by Fawzy and Bishop ${ }^{5}$ and Fawzy ${ }^{6}$ using second-order equations of motion. We define the normalization matrix $\Theta$ as

$$
\boldsymbol{\Theta}=\operatorname{diag}\left[\theta_{1}, \theta_{2}, \ldots, \theta_{m}\right] \in \mathbb{C}^{m \times m}
$$

Numerical values of $\theta_{k}$ can be selected in various ways:

1) Choose $\theta_{k}=2 \lambda_{k}, \forall k$, that is, $\Theta=2 \Lambda$. This reduces to $\boldsymbol{v}_{k}^{T} \boldsymbol{M} \boldsymbol{u}_{k}=1$ when the damping is zero. This is consistent with the unity modal mass convention, often used in experimental modal analysis and finite element methods.

2) Choose $\theta_{k}=1+0 i, \forall k$, that is, $\Theta=\boldsymbol{I}_{m}$. Theoretical analysis becomes easiest with this normalization. However, as pointed out by Fawzy ${ }^{6}$ and Vigneron ${ }^{7}$ in the context of viscously damped systems, this normalization is inconsistent with undamped or classically damped modal theories.

Finally, the normalization condition in Eq. (14) does not make the eigenvectors unique. Each right and left eigenvectors are still nonunique to the extent of unknown scalar multipliers. In other words, the matrices $\boldsymbol{U}$ and $\boldsymbol{V}$ are nonunique to the extent of $m \times m$ complex diagonal postmultiplier matrices.

\section{Orthogonality of the Eigenvectors}

The orthogonality relationship of the eigenvectors given by Eq. (10) is not very useful because it is expressed in terms of the eigenvalues of the system. In this section we will develop an orthogonality relationship that is independent of the eigenvalues. Expressions equivalent to the orthogonality relationships of the undamped eigenvectors with respect to the mass and stiffness matrices will also be established. To derive these results, the eigenvalue problems associated with the dynamic stiffness matrix and its inversion are considered first

\section{Eigensolutions of the Dynamic Stiffness Matrix}

For any given $s \in \mathbb{C}$ the right and left eigenvalue problems associated with the dynamic stiffness matrix can be expressed by

$$
\begin{array}{rlrl}
\boldsymbol{D}(s) \phi_{k}(s) & =v_{k}(s) \phi_{k}(s), & \forall k & =1, \ldots, N \\
\boldsymbol{\psi}_{k}^{T}(s) \boldsymbol{D}(s) & =v_{k}(s) \boldsymbol{\psi}_{k}^{T}(s), & \forall k=1, \ldots, N
\end{array}
$$

In these equations the eigenvalues $v_{k}(s) \in \mathbb{C}$ are the roots of the characteristic equation

$$
\operatorname{det}\left[\boldsymbol{D}(s)-v(s) \boldsymbol{I}_{N}\right]=0
$$


and $\phi_{k}(s), \psi_{k}(s) \in \mathbb{C}^{N}$ are respectively the $k$ th right and left eigenvectors of $\boldsymbol{D}(s)$. The symbols $v_{k}(s), \phi_{k}(s)$, and $\boldsymbol{\psi}_{k}(s)$ indicate functional dependence of these quantities on the complex parameter $s$. Such a continuous dependence is expected whenever $\boldsymbol{D}(s)$ is a sufficiently smooth matrix function of $s$. Because $\boldsymbol{D}(s)$ is an $N \times N$ complex matrix for a fixed $s$, the number of eigenvalues (and consequently the eigenvectors) must be $N$. Further, it can be shown that for distinct eigenvalues $\phi_{k}(s)$ and $\psi_{j}(s)$ also satisfy a biorthogonality relationship although $\boldsymbol{u}_{k}$ and $\boldsymbol{v}_{j}$ do not enjoy any such simple relationship. We normalize $\phi_{k}(s)$ and $\psi_{k}(s)$ such that

$$
\boldsymbol{\psi}_{j}^{T}(s) \phi_{k}(s)=\delta_{k j} \quad \forall k, j=1, \ldots, N
$$

In view of the preceding relationship, from Eqs. (16a) and (16b) and we have

$$
\boldsymbol{\psi}_{j}^{T}(s) \boldsymbol{D}(s) \phi_{k}(s)=v_{k}(s) \delta_{k j}, \quad \forall k, j=1, \ldots, N
$$

or in the matrix form

$$
\Psi^{T}(s) \boldsymbol{D}(s) \Phi(s)=\nu(s)
$$

Here

$$
\begin{gathered}
\Phi(s)=\left[\phi_{1}(s), \phi_{2}(s), \ldots, \phi_{N}(s)\right] \in \mathbb{C}^{N \times N} \\
\Psi(s)=\left[\boldsymbol{\psi}_{1}(s), \boldsymbol{\psi}_{2}(s), \ldots, \boldsymbol{\psi}_{N}(s)\right] \in \mathbb{C}^{N \times N} \\
\boldsymbol{\nu}(s)=\operatorname{diag}\left[v_{1}(s), v_{2}(s), \ldots, v_{N}(s)\right] \in \mathbb{C}^{N \times N}
\end{gathered}
$$

It is possible to establish the relationships between the original eigenvalue problems of the system defined by Eqs. (6a) and (6b) and that by Eqs. (16a) and (16b). Consider the case when the parameter $s$ approaches any one of the system eigenvalues, say $\lambda_{j}$. Because all of the $v_{k}(s)$ are assumed to be distinct, for nontrivial eigenvectors, comparing Eqs. (6a), (6b), (16a), and (16b) we can conclude that one and only one of the $v_{k}(s)$ must be zero ${ }^{8}$ when $s \rightarrow \lambda_{j}$. Suppose that the $r$ th eigenvalue of the eigenvalue problems (16a) and (16b) is zero when $s \rightarrow \lambda_{j}$. The eigenvectors in Eqs. (16a) and (16b) corresponding to the $r$ th eigenvalue also approach the eigenvectors in Eqs. (6a) and (6b) as $s \rightarrow \lambda_{j}$. Thus, when $s=\lambda_{j}$, one has

$$
\begin{gathered}
v_{r}\left(\lambda_{j}\right)=0, \quad v_{k}\left(\lambda_{j}\right) \neq 0, \quad \forall k=1, \ldots, N ; \neq r \\
\phi_{r}\left(\lambda_{j}\right)=\boldsymbol{u}_{j} \\
\boldsymbol{\psi}_{r}\left(\lambda_{j}\right)=\boldsymbol{v}_{j}
\end{gathered}
$$

These equations completely relate the eigensolutions of Eqs. (6a) and (6b) with that of Eqs. (16a) and (16b). Now, these relationships will be used to obtain the inverse of the dynamic stiffness matrix.

\section{Inversion of the Dynamic Stiffness Matrix}

The inverse of the dynamic stiffness matrix, often known as the transfer function matrix, can be expressed as

$$
\boldsymbol{D}^{-1}(s)=\frac{\operatorname{adj}[\boldsymbol{D}(s)]}{\operatorname{det}[\boldsymbol{D}(s)]} \in \mathbb{C}^{N \times N}
$$

The poles of $\boldsymbol{D}^{-1}(s)$ are exactly the eigenvalues of the system as given by Eq. (4). Because it is assumed that all of the $m$ eigenvalues are distinct, each pole is a simple pole. From the residue theorem we know that any complex function can be expressed in terms of the poles and residues, that is,

$$
\boldsymbol{D}^{-1}(s)=\sum_{j=1}^{m} \frac{\boldsymbol{R}_{\boldsymbol{j}}}{s-\lambda_{j}}
$$

Here

$$
\boldsymbol{R}_{j}=\underset{s=\lambda_{j}}{\operatorname{res}}\left[\boldsymbol{D}^{-1}(s)\right] \stackrel{\text { def }}{=} \lim _{s \rightarrow \lambda_{j}}\left(s-\lambda_{j}\right)\left[\boldsymbol{D}^{-1}(s)\right]
$$

is the residue of $\boldsymbol{D}^{-1}(s)$ at the pole $\lambda_{j}$.
Now, from Eq. (20) one has

$$
D^{-1}(s)=\Phi(s) \nu^{-1}(s) \Psi^{T}(s)
$$

Noting that $\boldsymbol{\nu}(s)$ is a diagonal matrix, we can expand the right-hand side of the preceding equation to obtain

$$
\boldsymbol{D}^{-1}(s)=\sum_{k=1}^{N} \frac{\phi_{k}(s) \boldsymbol{\psi}_{k}^{T}(s)}{v_{k}(s)}
$$

Separation of the $r$ th term in the preceding sum yields

$$
\boldsymbol{D}^{-1}(s)=\frac{\phi_{r}(s) \boldsymbol{\psi}_{r}^{T}(s)}{v_{r}(s)}+\left[\sum_{\substack{k=1 \\ k \neq r}}^{N} \frac{\phi_{k}(s) \boldsymbol{\psi}_{k}^{T}(s)}{v_{k}(s)}\right]
$$

Clearly, when $s \rightarrow \lambda_{j}$, the second term of the right-hand side of Eq. (32) is analytic because according to Eq. (24) $v_{k}\left(\lambda_{j}\right) \neq$ $0, \forall k=1, \ldots, N ; \neq r$. From Eq. (29) the residue at $s=\lambda_{j}$ can be obtained as

$$
\begin{aligned}
\boldsymbol{R}_{j} \stackrel{\text { def }}{=} \lim _{s \rightarrow \lambda_{j}}\left(s-\lambda_{j}\right)\left\{\frac{\phi_{r}(s) \psi_{r}^{T}(s)}{v_{r}(s)}+\left[\sum_{\substack{k=1 \\
k \neq r}}^{N} \frac{\phi_{k}(s) \psi_{k}^{T}(s)}{v_{k}(s)}\right]\right\} \\
=\lim _{s \rightarrow \lambda_{j}}\left(s-\lambda_{j}\right) \frac{\phi_{r}(s) \psi_{r}^{T}(s)}{v_{r}(s)}
\end{aligned}
$$

Using l'Hôspital's rule, the preceding expression can be rewritten as

$$
\boldsymbol{R}_{j}=\frac{\left.\phi_{r}(s) \boldsymbol{\psi}_{r}^{T}(s)\right|_{s=\lambda_{j}}}{\partial v_{r}(s) /\left.\partial s\right|_{s=\lambda_{j}}}+\lim _{s \rightarrow \lambda_{j}} \frac{\left(s-\lambda_{j}\right)(\partial / \partial s)\left[\phi_{k}(s) \boldsymbol{\psi}_{k}^{T}(s)\right]}{\partial v_{r}(s) / \partial s}
$$

It is clear that the second term of the right-hand side of the preceding equation is zero. Now, use of Eqs. (25) and (26) results in

$$
\boldsymbol{R}_{j}=\frac{\boldsymbol{u}_{j} \boldsymbol{v}_{j}^{T}}{\partial v_{r}(s) /\left.\partial s\right|_{s=\lambda_{j}}}
$$

The denominator in the preceding expression for the residues $\partial v_{r}(s) /\left.\partial s\right|_{s=\lambda_{j}}$ is still unknown. Consider the $r$ th right eigenvalue problem associated with the dynamic stiffness matrix. Differentiation of Eq. (16a) for $k=r$ with respect to $s$ yields

$$
\frac{\partial \boldsymbol{D}(s)}{\partial s} \boldsymbol{\phi}_{r}(s)+\boldsymbol{D}(s) \frac{\partial \phi_{r}(s)}{\partial s}=\frac{\partial v_{r}(s)}{\partial s} \boldsymbol{\phi}_{r}(s)+v_{r}(s) \frac{\partial \phi_{r}(s)}{\partial s}
$$

Premultiplying the preceding equation by $\boldsymbol{\psi}_{r}^{T}(s)$ and rearranging, one obtains

$$
\begin{aligned}
\boldsymbol{\psi}_{r}^{T}(s) & \frac{\partial \boldsymbol{D}(s)}{\partial s} \phi_{r}(s)+\left[\boldsymbol{\psi}_{r}^{T}(s) \boldsymbol{D}(s)-\boldsymbol{\psi}_{r}^{T}(s) v_{r}(s)\right] \frac{\partial \boldsymbol{\phi}_{r}(s)}{\partial s} \\
= & \boldsymbol{\psi}_{r}^{T}(s) \frac{\partial v_{r}(s)}{\partial s} \phi_{r}(s)
\end{aligned}
$$

From the definition of the left eigenvectors in Eq. (16b), it follows that the second term of the left-hand side of the preceding equation is zero. Using the normalization condition in Eq. (18) and letting $s \rightarrow \lambda_{j}$, from Eq. (37) we have

$$
\left.\frac{\partial v_{r}(s)}{\partial s}\right|_{s=\lambda_{j}}=\left.\boldsymbol{v}_{j}^{T} \frac{\partial \boldsymbol{D}(s)}{\partial s}\right|_{s=\lambda_{j}} \boldsymbol{u}_{j}=\boldsymbol{v}_{j}^{T} \boldsymbol{D}^{\prime}\left(\lambda_{j}\right) \boldsymbol{u}_{j}
$$

Interestingly, observe that the left-hand side of Eq. (38) is exactly the same as the normalization condition given by Eq. (14). Now, using Eqs. (14), (35), and (38) one finally obtains

$$
\boldsymbol{R}_{j}=\boldsymbol{u}_{j} \boldsymbol{v}_{j}^{T} / \theta_{j}
$$

Equation (39) completely relates the residues of $\boldsymbol{D}^{-1}(s)$ to the eigenvalues and eigenvectors of the system. 


\section{Orthogonality Relationships}

For undamped systems, i.e., when $\boldsymbol{G}(s)=\boldsymbol{O}_{N}, \forall s$, it is easy to show that the right and left eigenvectors $\boldsymbol{x}_{\boldsymbol{j}}$ and $\boldsymbol{y}_{\boldsymbol{j}}$ satisfy the biorthogonality relationship ${ }^{9}$ over the mass and stiffness matrices, that is,

$$
\begin{array}{r}
\boldsymbol{y}_{j}^{T} \boldsymbol{M} \boldsymbol{x}_{\boldsymbol{k}}=\delta_{\mathrm{jk}} \\
\boldsymbol{y}_{j}^{T} \boldsymbol{K} \boldsymbol{x}_{\boldsymbol{k}}=\omega_{j}^{2} \delta_{\mathrm{jk}}
\end{array}
$$

For viscously damped systems (with nonproportional damping) equivalent relationships can be obtained by converting the equations of motion into the state-space form. ${ }^{4}$ The eigenproblem in the state-space form is essentially similar to the undamped eigenproblem except that the size of the problem gets doubled and the eigensolutions become complex. Thus, from the analysis point of view state-space approach offers significant advantage for viscously damped systems. Unfortunately, for nonviscously damped systems no advantage can be gained by adopting the state-space formalism as at least one of the system matrices will not be a constant matrix. For this reason, and also realizing that the state-space eigenvectors are not physically appealing, we kept the analysis in the secondorder form. One of our main results regarding the orthogonality of the eigenvectors is the following:

Theorem 1: The matrices of the right and left eigenvectors of a nonviscously damped system $\boldsymbol{U}, \boldsymbol{V} \in \mathbb{C}^{N \times m}$ satisfy the orthogonality relationship $\boldsymbol{U} \Theta^{-1} \boldsymbol{V}^{T}=\boldsymbol{O}_{N}$.

Proof: From Eqs. (27), (28), and (39) one obtains

$$
\frac{\operatorname{adj}[\boldsymbol{D}(s)]}{\operatorname{det}[\boldsymbol{D}(s)]}=\sum_{j=1}^{m} \frac{1}{s-\lambda_{j}} \frac{\boldsymbol{u}_{j} \boldsymbol{v}_{j}^{T}}{\theta_{j}}
$$

Multiplying both sides of the preceding equation by $s$ and taking limit as $s \rightarrow \infty$, we obtain

$$
\lim _{s \rightarrow \infty} s \frac{\operatorname{adj}[\boldsymbol{D}(s)]}{\operatorname{det}[\boldsymbol{D}(s)]}=\lim _{s \rightarrow \infty} \sum_{j=1}^{m} \frac{s}{s-\lambda_{j}} \frac{\boldsymbol{u}_{j} \boldsymbol{v}_{j}^{T}}{\theta_{j}}=\sum_{j=1}^{m} \frac{\boldsymbol{u}_{j} \boldsymbol{v}_{j}^{T}}{\theta_{j}}
$$

It is easy to observe that the order of the elements of $\operatorname{adj}[\boldsymbol{D}(s)]$ is at the most $(m-2)$ in $s$. Because the order of the determinant $\operatorname{det}[\boldsymbol{D}(s)]$ is $m$, after taking the limit every element of the left-hand side of Eq. (43) reduces to zero. Thus, in the limit, the left-hand side of Eq. (43) approaches to an $N \times N$ null matrix. Finally, writing Eq. (43) in the matrix form we obtain

$$
\boldsymbol{U} \Theta^{-1} \boldsymbol{V}^{T}=\boldsymbol{O}_{N}
$$

and the theorem is proved.

The result of this theorem is quite general, and it does not depend on the nature of the system property matrices. The only requirement of this theorem is that the system must have $m \geq 2 N$ distinct eigenvalues. Clearly, the undamped systems as well as the viscously damped systems are also covered as special cases. In the context of viscously damped systems (see the Appendix for details), a similar result has been derived by Fawzy and Bishop ${ }^{5}$ by considering the normalization matrix $\Theta$ as the identity matrix. Later Fawzy ${ }^{6}$ generalized this result for the case when $\Theta$ is not an identity matrix. The result obtained in theorem 1 can be viewed as a further generalization of these results to nonviscously damped systems. Next we consider the relationship between the eigensolutions and the mass matrix.

Theorem 2: The matrices of the right and left eigenvectors of a nonviscously damped system $\boldsymbol{U}, \boldsymbol{V} \in \mathbb{C}^{N \times m}$ satisfy the relationship $\boldsymbol{U} \Theta^{-1} \Lambda \boldsymbol{V}^{T}=\boldsymbol{M}^{-1}$.

Proof: First consider the function $s \boldsymbol{D}^{-1}(s)$. Following the approach outlined in the last section and using the residue theorem, one obtains

$$
s \boldsymbol{D}^{-1}(s)=\sum_{j=1}^{m} \frac{\boldsymbol{Q}_{j}}{s-\lambda_{j}}
$$

Here the residues $\boldsymbol{Q}_{j}$ can be obtained as

$$
\boldsymbol{Q}_{j} \stackrel{\text { def }}{=} \lim _{s \rightarrow \lambda_{j}}\left(s-\lambda_{j}\right)\left[s \boldsymbol{D}^{-1}(s)\right]=\lambda_{j}\left(\boldsymbol{u}_{j} \boldsymbol{v}_{j}^{T} / \theta_{j}\right)
$$

Using the expression of the dynamic stiffness matrix in Eq. (3), we can deduce

$$
\lim _{s \rightarrow \infty}\left[\boldsymbol{D}(s) / s^{2}\right]=\lim _{s \rightarrow \infty}\left\{\boldsymbol{M}+[\boldsymbol{G}(s) / s]+\left(\boldsymbol{K} / s^{2}\right)\right\}=\boldsymbol{M}
$$

Taking the inverse of the preceding equation results in

$$
\lim _{s \rightarrow \infty}\left[s^{2} \boldsymbol{D}^{-1}(s)\right]=\boldsymbol{M}^{-1}
$$

Now, multiplying Eq. (45) by $s$ and taking limit as $s \rightarrow \infty$, we obtain

$$
\lim _{s \rightarrow \infty}\left[s^{2} \boldsymbol{D}^{-1}(s)\right]=\lim _{s \rightarrow \infty} \sum_{j=1}^{m} \frac{s}{s-\lambda_{j}} \frac{\lambda_{j} \boldsymbol{u}_{j} \boldsymbol{v}_{j}^{T}}{\theta_{j}}=\sum_{j=1}^{m} \frac{\lambda_{j} \boldsymbol{u}_{j} \boldsymbol{v}_{j}^{T}}{\theta_{j}}
$$

Casting the right-hand side of the preceding equation in the matrix form and equating it with Eq. (48) results in

$$
\boldsymbol{U} \Theta^{-1} \Lambda V^{T}=M^{-1}
$$

and the theorem is proved.

Because $\Theta$ and $\Lambda$ are diagonal matrices, they commute in product. For this reason the preceding result can also be expressed as $\boldsymbol{U} \Lambda \Theta^{-1} \boldsymbol{V}^{T}=\boldsymbol{M}^{-1}$. For viscously damped systems a similar result has been derived by Fawzy and Bishop ${ }^{5}$ by considering the normalization matrix $\Theta$ as the identity matrix. It might be thought that by taking the inverse of Eq. (50) and rearranging the conventional mass-orthogonality relationship

$$
\boldsymbol{V}^{T} \boldsymbol{M U}=\Lambda^{-1} \Theta
$$

could be obtained. We emphasize that the representation of Eq. (50) in the form of Eq. (51) is not always possible. To show this, premultiply Eq. (51) by $\boldsymbol{U} \Theta^{-1}$ to obtain

$$
\boldsymbol{U} \Theta^{-1} \boldsymbol{V}^{T} \boldsymbol{M} \boldsymbol{U}=\boldsymbol{U} \Theta^{-1} \Lambda^{-1} \Theta=\boldsymbol{U} \Lambda^{-1}
$$

Because of theorem 1, the left-hand side of Eq. (52) is a null matrix, whereas its right-hand side is not. Thus Eq. (51) cannot be a valid equation. However, for a special case, when the system is undamped, the modal matrices $\boldsymbol{U}$ and $\boldsymbol{V}$ can be expressed by square matrices, and Eq. (50) can be represented by the classical massorthogonality relationship in Eq. (51). Thus, theorem 2 provides the result equivalent to the classical mass-orthogonality relationship for general cases.

Like the mass-orthogonality relationship of the eigenvectors, the orthogonality relationship with respect to the stiffness matrix can also be obtained. Assuming that $\boldsymbol{K}^{-1}$ exists, we have the following theorem and proof.

Theorem 3: The matrices of the right and left eigenvectors of a nonviscously damped system $\boldsymbol{U}, \boldsymbol{V} \in \mathbb{C}^{N \times m}$ satisfy the relationship $\boldsymbol{U} \Theta^{-1} \Lambda^{-1} \boldsymbol{V}^{T}=-\boldsymbol{K}^{-1}$.

Proof: Using the expression of the dynamic stiffness matrix in Eq. (3), we can easily deduce

$$
\lim _{s \rightarrow 0} \boldsymbol{D}(s)=\boldsymbol{K}
$$

Taking the inverse of the preceding equation results in

$$
\lim _{s \rightarrow 0} \boldsymbol{D}^{-1}(s)=\boldsymbol{K}^{-1}
$$

From Eqs. (28) and (29) one obtains

$$
\boldsymbol{D}^{-1}(s)=\sum_{j=1}^{m} \frac{1}{s-\lambda_{j}} \frac{\boldsymbol{u}_{j} \boldsymbol{v}_{j}^{T}}{\theta_{j}}
$$

Taking the limit as $s \rightarrow 0$ in Eq. (55), we obtain

$$
\lim _{s \rightarrow 0} \boldsymbol{D}^{-1}(s)=\sum_{j=1}^{m} \frac{1}{-\lambda_{j}} \frac{\boldsymbol{u}_{j} \boldsymbol{v}_{j}^{T}}{\theta_{j}}
$$


Casting the right-hand side of the preceding equation in the matrix form and equating it with Eq. (54) results in

$$
\boldsymbol{U} \Theta^{-1} \Lambda^{-1} \boldsymbol{V}^{T}=-\boldsymbol{K}^{-1}
$$

and the theorem is proved.

\section{Relationships Between the Eigensolutions and Damping}

In the last section some direct relationships have been established between the mass and stiffness matrices and the eigensolutions. In this section we try to establish the relationships between the damping matrix and the eigensolutions. A major difficulty in this regard is that, unlike the mass and stiffness matrices, the damping matrix $\boldsymbol{G}(s)$ is a function of $s$. To simplify the problem, we consider only two limiting cases: 1) when $s \rightarrow \infty$ and 2) when $s \rightarrow 0$. Suppose

$$
\begin{gathered}
\lim _{s \rightarrow \infty} \boldsymbol{G}(s)=\boldsymbol{G}_{\infty} \in \mathbb{R}^{N \times N} \\
\lim _{s \rightarrow 0} \boldsymbol{G}(s)=\boldsymbol{G}_{0} \in \mathbb{R}^{N \times N}
\end{gathered}
$$

where $\left\|\boldsymbol{G}_{\infty}\right\|,\left\|\boldsymbol{G}_{0}\right\|<\infty$.

\section{Relationships in Terms of $M^{-1}$}

Casting Eq. (55) into the matrix form, one obtains

$$
\boldsymbol{D}^{-1}(s)=\boldsymbol{U} \Theta^{-1}\left(s \boldsymbol{I}_{m}-\Lambda\right)^{-1} \boldsymbol{V}^{T}
$$

The preceding equation can be expanded as

$$
\begin{aligned}
\boldsymbol{D}^{-1}( & s)=(1 / s) \boldsymbol{U} \Theta^{-1}\left(\boldsymbol{I}_{m}-\Lambda / s\right)^{-1} \boldsymbol{V}^{T} \\
= & (1 / s)\left(\boldsymbol{U} \Theta^{-1} \boldsymbol{V}^{T}\right)+\left(1 / s^{2}\right)\left(\boldsymbol{U} \Theta^{-1} \Lambda \boldsymbol{V}^{T}\right) \\
+ & \left(1 / s^{3}\right)\left(\boldsymbol{U} \Theta^{-1} \Lambda^{2} \boldsymbol{V}^{T}\right)+\left(1 / s^{4}\right)\left(\boldsymbol{U} \Theta^{-1} \Lambda^{3} \boldsymbol{V}^{T}\right)+\cdots
\end{aligned}
$$

Now rewrite the expression of the dynamic stiffness matrix in Eq. (3) as

$$
\boldsymbol{D}(s)=s^{2} \boldsymbol{M}\left\{\boldsymbol{I}_{N}+\left(\boldsymbol{M}^{-1} / s\right)[\boldsymbol{G}(s)+\boldsymbol{K} / s]\right\}
$$

Taking the inverse of this equation and expanding the right-hand side, one obtains

$$
\begin{aligned}
\boldsymbol{D}^{-1}(s) & =\left(\boldsymbol{I}_{N}-\left(\boldsymbol{M}^{-1} / s\right)\{\boldsymbol{G}(s)+(\boldsymbol{K} / s)\}\right. \\
+ & \left.\left\{\boldsymbol{M}^{-1} / s[\boldsymbol{G}(s)+(\boldsymbol{K} / s)]\right\}^{2}-\cdots\right) \boldsymbol{M}^{-1} / s^{2}
\end{aligned}
$$

Equation (63) can be further simplified to obtain

$$
\begin{aligned}
& \boldsymbol{D}^{-1}(s)=\left(\boldsymbol{M}^{-1} / s^{2}\right)+\left(1 / s^{3}\right)\left[-\boldsymbol{M}^{-1} \boldsymbol{G}(s) \boldsymbol{M}^{-1}\right] \\
& +\left(1 / s^{4}\right)\left\{\boldsymbol{M}^{-1}\left[\boldsymbol{G}(s) \boldsymbol{M}^{-1} \boldsymbol{G}(s)-\boldsymbol{K}\right] \boldsymbol{M}^{-1}\right\}+\cdots
\end{aligned}
$$

Comparing Eqs. (61) and (64), it is clear that their right-hand sides are equal. Theorems 1 and 2 can be alternatively proved by multiplying these equations by $s$ and $s^{2}$ respectively and taking the limit as $s \rightarrow \infty$. Now multiply Eqs. (61) and (64) by $s^{3}$ and take the limit as $s \rightarrow \infty$. The matrix coefficients associated with the lower-order powers of $s$ are effected by the functional variation of $\boldsymbol{G}(s)$ from the preceding terms because in the limit all of the later terms would be zero. Because the coefficient matrix associated with $1 / s^{3}$ of the right-hand side of Eq. (64) is the first term containing $\boldsymbol{G}(s)$, only this term can be equated with the corresponding term (the third term) of the right-hand side of Eq. (61). Thus taking the limit as $s \rightarrow \infty$, one obtains

$$
\boldsymbol{U} \Theta^{-1} \Lambda^{2} \boldsymbol{V}^{T}=-\boldsymbol{M}^{-1} \boldsymbol{G}_{\infty} \boldsymbol{M}^{-1}
$$

This procedure cannot be extended to equate the coefficient matrices associated with further lower order powers of $s$ as all of them would be affected by the functional variation of $\boldsymbol{G}(s)$ from preceding terms.

Were the system viscously damped, $\boldsymbol{G}(s)$ would be a constant matrix, and equating the coefficient matrices associated with different powers of $s$ one could obtain several relationships between the eigensolutions and the system matrices. Considering the first few terms in the series expressions (61) and (64), some such relationships are reported in the Appendix.

\section{Relationships in Terms of $K^{-1}$}

We rewrite Eq. (60) as

$$
\boldsymbol{D}^{-1}(s)=-\boldsymbol{U} \Theta^{-1} \Lambda^{-1}\left(\boldsymbol{I}_{m}-s \Lambda^{-1}\right)^{-1} \boldsymbol{V}^{T}
$$

Expanding Eq. (66), one obtains

$$
\begin{aligned}
& \boldsymbol{D}^{-1}(s)=-\boldsymbol{U} \Theta^{-1} \Lambda^{-1} \boldsymbol{V}^{T}-s\left(\boldsymbol{U} \Theta^{-1} \Lambda^{-2} \boldsymbol{V}^{T}\right) \\
& \quad-s^{2}\left(\boldsymbol{U} \Theta^{-1} \Lambda^{-3} \boldsymbol{V}^{T}\right)-s^{3}\left(\boldsymbol{U} \Theta^{-1} \Lambda^{-4} \boldsymbol{V}^{T}\right)-\cdots
\end{aligned}
$$

The expression of the dynamic stiffness matrix in Eq. (3) can be rearranged as

$$
\boldsymbol{D}(s)=\boldsymbol{K}\left\{\boldsymbol{I}_{N}+s\left[s \boldsymbol{K}^{-1} \boldsymbol{M}+\boldsymbol{K}^{-1} \boldsymbol{G}(s)\right]\right\}
$$

Taking the inverse of Eq. (68) and expanding the right-hand side, one obtains

$$
\begin{aligned}
& \boldsymbol{D}^{-1}(s)=\left(\boldsymbol{I}_{N}-s\left[s \boldsymbol{K}^{-1} \boldsymbol{M}+\boldsymbol{K}^{-1} \boldsymbol{G}(s)\right]\right. \\
& \left.+\left\{s\left[s \boldsymbol{K}^{-1} \boldsymbol{M}+\boldsymbol{K}^{-1} \boldsymbol{G}(s)\right]\right\}^{2}-\cdots\right) \boldsymbol{K}^{-1}
\end{aligned}
$$

The preceding equation can be further simplified to obtain

$$
\begin{aligned}
& \boldsymbol{D}^{-1}(s)=\boldsymbol{K}^{-1}+s\left[-\boldsymbol{K}^{-1} \boldsymbol{G}(s) \boldsymbol{K}^{-1}\right] \\
& \quad+s^{2}\left\{\boldsymbol{K}^{-1}\left[\boldsymbol{G}(s) \boldsymbol{K}^{-1} \boldsymbol{G}(s)-\boldsymbol{M}\right] \boldsymbol{K}^{-1}\right\}+\cdots
\end{aligned}
$$

Comparing the right-hand side of Eqs. (67) and (70), theorem 3 can be proved alternatively by taking the limit as $s \rightarrow 0$. Considering the second term of the right-hand side of Eq. (70), equating it with the second term of Eq. (67), and taking the limit as $s \rightarrow 0$, one obtains

$$
\boldsymbol{U} \Theta^{-1} \Lambda^{-2} \boldsymbol{V}^{T}=\boldsymbol{K}^{-1} \boldsymbol{G}_{0} \boldsymbol{K}^{-1}
$$

Again, this approach cannot be extended to equate the coefficient matrices associated with further higher-order powers of $s$ as all of them would be affected by the functional variation of $\boldsymbol{G}(s)$ from preceding terms.

Theorems 2 and 3 and Eqs. (65) and (71) allow us to represent the system property matrices explicitly in terms of the eigensolutions. This might be useful in system identification problems where the eigensolutions of a structure can be measured from experiments. Using the eigensolutions, we define two matrices:

$$
\begin{gathered}
\boldsymbol{P}_{1}=\boldsymbol{U} \Theta^{-1} \Lambda \boldsymbol{V}^{T} \\
\boldsymbol{P}_{2}=\boldsymbol{U} \Theta^{-1} \Lambda^{-1} \boldsymbol{V}^{T}
\end{gathered}
$$

Using these equations, from Eq. (50) one obtains the mass matrix as

$$
\boldsymbol{M}=\boldsymbol{P}_{1}^{-1}
$$

Similarly, from Eq. (57) the stiffness matrix can be obtained as

$$
\boldsymbol{K}=-\boldsymbol{P}_{2}^{-1}
$$

The damping matrix in the Laplace domain $\boldsymbol{G}(s)$ can be obtained only at the two limiting values when $s \rightarrow \infty$ and $s \rightarrow 0$. From Eqs. (65) and (71) one obtains

$$
\begin{aligned}
\boldsymbol{G}_{\infty} & =-\boldsymbol{P}_{1}^{-1}\left[\boldsymbol{U} \Theta^{-1} \Lambda^{2} \boldsymbol{V}^{T}\right] \boldsymbol{P}_{1}^{-1} \\
\boldsymbol{G}_{0} & =\boldsymbol{P}_{2}^{-1}\left[\boldsymbol{U} \Theta^{-1} \Lambda^{-2} \boldsymbol{V}^{T}\right] \boldsymbol{P}_{2}^{-1}
\end{aligned}
$$

These results, however, do not give any indication regarding the functional behavior of $\boldsymbol{G}(s)$ between these two extreme values. 


\section{Numerical Examples}

System

A three-degree-of-freedom asymmetric system is considered to illustrate the results derived in this paper. The mass and stiffness matrices are assumed to be

$$
\begin{gathered}
\boldsymbol{M}=\left[\begin{array}{rrr}
0.5740 & 1.3858 & 1.3858 \\
0.7070 & 0.7070 & -0.7070 \\
0.4620 & -0.1914 & -0.1914
\end{array}\right] \\
\boldsymbol{K}=\left[\begin{array}{rrr}
1.3748 & 10.9440 & 25.2975 \\
1.2625 & 2.8770 & -17.4195 \\
0.7455 & -4.1244 & 0.8625
\end{array}\right]
\end{gathered}
$$

Numerical values for these matrices are taken from Adhikari. ${ }^{10}$ The matrix of the damping functions is assumed to be of the form

$$
\mathcal{G}(t)=\left[\begin{array}{rrr}
2.6710 \delta(t) & 2.9592 \delta(t) & 2.9651 \delta(t) \\
0.4843 \delta(t) & 1.2606 g(t) & -0.6119 \delta(t) \\
0.2875 \delta(t) & -0.5808 \delta(t) & 1.2272 \delta(t)
\end{array}\right]
$$

where

$$
g(t)=\delta(t)+\left(\mu_{1} e^{-\mu_{1} t}+\mu_{2} e^{-\mu_{2} t}\right), \quad \quad \mu_{1}, \mu_{2}>0
$$

The damping matrix in the Laplace domain $\boldsymbol{G}(s)$ can be obtained by taking the Laplace transform of Eq. (80). The Laplace transform of $g(t)$ given by Eq. (81) can be obtained as

$$
G(s)=1+\frac{\left(\mu_{1}+\mu_{2}\right) s+2 \mu_{1} \mu_{2}}{s^{2}+\left(\mu_{1}+\mu_{2}\right) s+\mu_{1} \mu_{2}}
$$

This damping model is a linear combination of the viscous model and the GHM model. ${ }^{2,11}$ Regarding the numerical values of the damping parameters, we assume $\mu_{1}=1$ and $\mu_{2}=2$.

\section{Eigenvalues and Eigenvectors}

Using Eq. (82), together with the expressions of the system matrices given by Eqs. (78-80), it can be shown that the order of the characteristic polynomial $m=8$. It has been mentioned that (for lightly damped systems), among the $m$ eigenvalues, $2 N=6$ appear in complex conjugate pairs (elastic modes) and the rest $p=m-2 N=2$ eigenvalues become purely real (nonviscous modes).

Solving the characteristic equation, the diagonal matrix containing the eigenvalues can be expressed as

$$
\Lambda=\operatorname{diag}\left[\boldsymbol{\lambda}_{e}, \boldsymbol{\lambda}_{e}^{*}, \boldsymbol{\lambda}_{n}\right] \in \mathbb{C}^{8 \times 8}
$$

Here, the eigenvalues corresponding to the three elastic modes are $\lambda_{e}=$

$\{-0.7666+1.3038 i, \quad-0.7628+3.0541 i, \quad-1.5489+4.5701 i\}$

The eigenvalues corresponding to the two nonviscous modes are found to be

$$
\lambda_{n}=\{-1.7285,-0.8998\}
$$

Because these eigenvalues are purely real and negative, it implies that the nonviscous modes are stable and nonoscillatory in nature (i.e., overcritically damped).

The eigenvectors can be obtained from Eqs. (6a) and (6b) by fixing any one element and inverting the associated matrix. The matrices of the right and left eigenvectors can be expressed as

$$
\begin{aligned}
& \boldsymbol{U}=\left[\boldsymbol{U}_{e}, \boldsymbol{U}_{e}^{*}, \boldsymbol{U}_{n}\right] \in \mathbb{C}^{3 \times 8} \\
& \boldsymbol{V}=\left[\boldsymbol{V}_{e}, \boldsymbol{V}_{e}^{*}, \boldsymbol{V}_{n}\right] \in \mathbb{C}^{3 \times 8}
\end{aligned}
$$

Here $\boldsymbol{U}_{e}$ and $\boldsymbol{V}_{e}$, the matrices of the right and left eigenvectors corresponding to the three elastic modes, are

$\boldsymbol{U}_{e}=$

$$
\left[\begin{array}{ccc}
1.0000 & 1.0000 & 1.0000 \\
0.0273-0.1400 i & -1.2745-0.7317 i & -1.0101+0.4615 i \\
0.0415-0.0657 i & -0.1475-0.3960 i & 0.8509-0.7761 i
\end{array}\right]
$$

$$
\begin{aligned}
& V_{e}= \\
& {\left[\begin{array}{ccc}
1.0000 & 1.0000 & 1.0000 \\
1.1553+0.4585 i & 0.0754+0.0686 i & -2.1760+0.0719 i \\
2.7274+1.4597 i & -1.0436+1.7964 i & 2.3613+0.9464 i
\end{array}\right] }
\end{aligned}
$$

Eigenvectors corresponding to the two nonviscous modes can be calculated as

$\boldsymbol{U}_{n}=\left[\begin{array}{cc}1.0000 & 1.0000 \\ 0.5146 & 0.2365 \\ -0.1482 & -0.0699\end{array}\right], \quad \boldsymbol{V}_{n}=\left[\begin{array}{cc}1.0000 & 1.0000 \\ 1.4464 & 1.3986 \\ -1.3160 & -1.6181\end{array}\right]$

Using the eigenvectors given by Eqs. (88-90), the normalization matrix $\Theta$ can be obtained from Eq. (13) as

$$
\Theta=\operatorname{diag}\left[\boldsymbol{\theta}_{e}, \boldsymbol{\theta}_{e}^{*}, \boldsymbol{\theta}_{n}\right]
$$

Here

$\boldsymbol{\theta}_{e}=$

$\{-1.9633+5.6936 i, \quad 4.5186-16.5636 i, \quad 17.7940+26.2194 i\}$

$\boldsymbol{\theta}_{n}=\{50.6436,44.6250\}$

\section{Orthogonality Relationships}

Using $\boldsymbol{U}, \boldsymbol{V}$, and $\Theta$, one can easily verify that the mode orthogonality relationship given by theorem 1 is satisfied, that is,

$$
\boldsymbol{U} \Theta^{-1} \boldsymbol{V}^{T}=\boldsymbol{O}_{3}
$$

Now, in the line of theorem 2 we calculate

$$
\boldsymbol{U} \Theta^{-1} \Lambda \boldsymbol{V}^{T}=\left[\begin{array}{rrr}
0.2552 & 0.0000 & 1.8475 \\
0.1804 & 0.7072 & -1.3064 \\
0.4355 & -0.7072 & 0.5411
\end{array}\right]=\boldsymbol{M}^{-1}
$$

Similarly, following theorem 3 one obtains

$$
-\boldsymbol{U} \Theta^{-1} \Lambda^{-1} \boldsymbol{V}^{T}=\left[\begin{array}{rrr}
0.1593 & 0.2613 & 0.6050 \\
0.0323 & 0.0406 & -0.1284 \\
0.0169 & -0.0318 & 0.0227
\end{array}\right]=\boldsymbol{K}^{-1}
$$

\section{Relationships with the Damping Matrix}

Taking the Laplace transform of Eq. (80) and considering the limiting cases as $s \rightarrow \infty$ and $s \rightarrow 0$, one obtains

$$
\begin{gathered}
\boldsymbol{G}_{\infty}=\left[\begin{array}{rrr}
2.6710 & 2.9592 & 2.9651 \\
0.4843 & 1.2606 & -0.6119 \\
0.2875 & -0.5808 & 1.2272
\end{array}\right] \\
\boldsymbol{G}_{0}=\left[\begin{array}{rrr}
2.6710 & 2.9592 & 2.9651 \\
0.4843 & 3.7818 & -0.6119 \\
0.2875 & -0.5808 & 1.2272
\end{array}\right]
\end{gathered}
$$


The matrices $\boldsymbol{P}_{1}$ and $\boldsymbol{P}_{2}$, defined in Eqs. (72) and (73), respectively, can be directly obtained from Eqs. (95) and (96). Using these matrices, the truth of Eqs. (76) and (77), which relate $\boldsymbol{G}_{\infty}$ and $\boldsymbol{G}_{0}$ to the eigensolutions, can be verified. Thus, the damping matrix $\boldsymbol{G}(s)$ can be reconstructed from the eigensolutions for the cases when $s \rightarrow \infty$ and $s \rightarrow 0$.

\section{Conclusions}

In this paper we have examined nonviscously damped multipledegree-of-freedom linear dynamic systems. The nonviscous damping model is such that the damping forces depend on the history of motion via convolution integrals over some kernel functions. The familiar viscous damping model is a special case of this general linear damping model when the kernel functions have no memory. For the sake of generality, neither is it assumed that the system matrices are symmetric nor is it assumed that they can be simultaneously diagonalized so that the classical modal analysis can be applied. The analysis is, however, restricted to systems with nonrepetitive eigenvalues.

Relationships regarding the normalization and the orthogonality of the (complex) right and left eigenvectors have been established (theorem 1). Expressions equivalent to the orthogonality of the undamped modes over the mass and stiffness matrices have been proposed (theorems 2 and 3). It was shown that the classical relationships can be obtained as special cases of these general results. Based on these results, we have shown that the mass and stiffness matrices can be uniquely expressed in terms of the eigensolutions. The damping matrix $\boldsymbol{G}(s)$ cannot be reconstructed using this approach because it is not a constant matrix. However, we have provided expressions that relate the damping matrix to the eigensolution for the cases when $s \rightarrow \infty$ and $s \rightarrow 0$. Whenever applicable, viscously damped counterparts of the newly developed results were provided.

\section{Appendix: Eigenrelations for Viscously Damped Systems}

Viscously damped systems arise as a special case of the more general nonviscously damped systems when the damping matrix become a constant matrix, that is, $\boldsymbol{G}(s)=\boldsymbol{C} \in \mathbb{R}^{N \times N}, \forall s$. Here, several relationships satisfied by the eigensolutions and the system matrices will be derived for this special case.

For viscously damped systems the order of the characteristic polynomial $m=2 N$, and consequently the modal matrices $\boldsymbol{U}, \boldsymbol{V} \in \mathbb{C}^{N \times 2 N}$ and the diagonal matrices $\Lambda, \Theta \in \mathbb{C}^{2 N \times 2 N}$. From Eq. (13) the normalization relationship reads

$$
\boldsymbol{v}_{k}^{T}\left[2 \lambda_{k} \boldsymbol{M}+\boldsymbol{C}\right] \boldsymbol{u}_{k}=\theta_{k}, \quad \forall k=1, \ldots, 2 N
$$

Now consider the series expansion of $\boldsymbol{D}^{-1}(s)$ given by Eqs. (61) and (64). Equating the coefficients of $1 / s$, we obtain the mode orthogonality relationship

$$
\boldsymbol{U} \Theta^{-1} \boldsymbol{V}^{T}=\boldsymbol{O}_{N}
$$

This relationship was also derived by Fawzy. ${ }^{6}$ Now, equating the coefficient matrices of $1 / s^{2}, \ldots, 1 / s^{5}$ on the right-hand sides of Eqs. (61) and (64), several relationships involving the eigensolutions and $\boldsymbol{M}^{-1}, \boldsymbol{C}$, and $\boldsymbol{K}$ can be obtained:

$$
\begin{gathered}
\boldsymbol{U} \Theta^{-1} \Lambda \boldsymbol{V}^{T}=\boldsymbol{M}^{-1} \\
\boldsymbol{U} \Theta^{-1} \Lambda^{2} \boldsymbol{V}^{T}=-\boldsymbol{M}^{-1} \boldsymbol{C} \boldsymbol{M}^{-1} \\
\boldsymbol{U} \Theta^{-1} \Lambda^{3} \boldsymbol{V}^{T}=\boldsymbol{M}^{-1}\left[\boldsymbol{C} \boldsymbol{M}^{-1} \boldsymbol{C}-\boldsymbol{K}\right] \boldsymbol{M}^{-1}
\end{gathered}
$$

$$
\boldsymbol{U} \Theta^{-1} \Lambda^{4} \boldsymbol{V}^{T}=\boldsymbol{M}^{-1}\left[\boldsymbol{K} \boldsymbol{M}^{-1} \boldsymbol{C}+\boldsymbol{C} \boldsymbol{M}^{-1} \boldsymbol{K}-\boldsymbol{C} \boldsymbol{M}^{-1} \boldsymbol{C} \boldsymbol{M}^{-1} \boldsymbol{C}\right] \boldsymbol{M}^{-1}
$$

This procedure can be extended to obtain further higher-order terms involving $\Lambda$.

Similarly, equating the coefficient matrices of $s^{0}, \ldots, s^{3}$ in the right-hand sides of Eqs. (67) and (70), several relationships involving the eigensolutions and $\boldsymbol{K}^{-1}, \boldsymbol{C}$, and $\boldsymbol{M}$ can be obtained:

$$
\begin{gathered}
\boldsymbol{U} \Theta^{-1} \Lambda^{-1} \boldsymbol{V}^{T}=-\boldsymbol{K}^{-1} \\
\boldsymbol{U} \Theta^{-1} \Lambda^{-2} \boldsymbol{V}^{T}=\boldsymbol{K}^{-1} \boldsymbol{C} \boldsymbol{K}^{-1} \\
\boldsymbol{U} \Theta^{-1} \Lambda^{-3} \boldsymbol{V}^{T}=\boldsymbol{K}^{-1}\left[\boldsymbol{M}-\boldsymbol{C} \boldsymbol{K}^{-1} \boldsymbol{C}\right] \boldsymbol{K}^{-1} \\
\boldsymbol{U} \Theta^{-1} \Lambda^{-4} \boldsymbol{V}^{T}=\boldsymbol{K}^{-1}\left[\boldsymbol{C} \boldsymbol{K}^{-1} \boldsymbol{C} \boldsymbol{K}^{-1} \boldsymbol{C}-\boldsymbol{M} \boldsymbol{K}^{-1} \boldsymbol{C}+\boldsymbol{C} \boldsymbol{K}^{-1} \boldsymbol{M}\right] \boldsymbol{K}^{-1}
\end{gathered}
$$

This procedure can be extended to obtain further lower-order terms involving $\Lambda$. Employing a different approach, and considering the normalization matrix $\Theta$ as the identity matrix, Fawzy and Bishop ${ }^{5}$ obtained expressions similar to Eqs. (A3-A5) and (A7-A9). Thus, the relationships derived here extend their results to generally normalized eigenvectors.

\section{Acknowledgments}

The author is grateful for the financial support provided by the Nehru Memorial Trust, London, and the Cambridge Commonwealth Trust.

\section{References}

${ }^{1}$ Adhikari, S., "Damping Models for Structural Vibration," Ph.D. Dissertation, Engineering Dept., Cambridge Univ., Cambridge, England, U.K., Sept. 2000.

${ }^{2}$ Golla, D. F., and Hughes, P. C., "Dynamics of Viscoelastic Structures-A Time Domain Finite Element Formulation," Journal of Applied Mechanics, Vol. 52, Dec. 1985, pp. 897-906.

${ }^{3}$ Lancaster, P., Lambda-Matrices and Vibrating System, Pergamon, London, 1966.

${ }^{4}$ Sestieri, A., and Ibrahim, R., "Analysis of Errors and Approximation s in the Use of Modal Coordinates," Journal of Sound and Vibration, Vol. 177, No. 2, 1994, pp. 145-157.

${ }^{5}$ Fawzy, I., and Bishop, R. E. D., "On the Dynamics of Linear NonConservative Systems," Proceeding s of the Royal Society of London, Series A, Vol. 352, 1976, pp. 25-40.

${ }^{6}$ Fawzy, I., "Orthogonality of Generally Normalized Eigenvectors and Eigenrows," AIAA Journal, Vol. 15, No. 2, 1977, pp. 276-278.

${ }^{7}$ Vigneron, F. R., "A Natural Modes Model and Modal Identities for Damped Linear Structures," Journal of Applied Mechanics, Vol. 53, March 1986, pp. 33-38.

${ }^{8}$ Yang, B., and Wu, X., "Modal Expansion of Structural Systems with Time Delays," AIAA Journal, Vol. 36, No. 12, 1998, pp. 2218-2224.

${ }^{9}$ Huseyin, K., Vibration and Stability of Multiple Parameter Systems, Sijhoff and Noordhoff, Alphen anden Rijn, The Netherlands, 1978.

${ }^{10}$ Adhikari, S., "Modal Analysis of Linear Asymmetric Non-Conservative Systems," Journal of Engineering Mechanics, Vol. 125, No. 12, 1999, pp. $1372-1379$.

${ }^{11}$ McTavis, D. J., and Hughes, P. C., "Modeling of Linear Viscoelastic Space Structures," Journal of Vibration and Acoustics, Vol. 115, Jan. 1993, pp. $103-110$.
E. R. Johnson Associate Editor 